\title{
A Vein-Viewing Application Enabled Detecting Abdominal Wall Varices Related to the Presence of Non-Treated Gastroesophageal Varices: A Cross- Sectional Study
}

\author{
Yoshiki Hoshino \\ Tottori University \\ Takaaki Sugihara ( $\sim$ sugitaka@tottori-u.ac.jp ) \\ Tottori University
}

Suguru Ikeda

Tottori University

Yukako Matsuki

Tottori University

Takakazu Nagahara

Tottori University

Jun-ichi Okano

Tottori University

Hajime Isomoto

Tottori University

\section{Research Article}

Keywords: abdominal wall varices, cirrhosis, gastroesophageal varices

Posted Date: April 14th, 2021

DOI: https://doi.org/10.21203/rs.3.rs-411097/v1

License: (c) (1) This work is licensed under a Creative Commons Attribution 4.0 International License.

Read Full License

Version of Record: A version of this preprint was published at BMC Medical Imaging on August 9th, 2021. See the published version at https://doi.org/10.1186/s12880-021-00655-8. 


\section{Abstract \\ Background}

Gastroesophageal varices (GOV) are a life-threatening complication in chronic liver disease. A method for non-invasively predicting GOV is crucial for management. This study aimed to determine whether a veinviewing application can detect abdominal wall varices (AWV) and elucidate the relationship between AWV and GOV.

\section{Methods}

One-hundred patients with chronic liver diseases were prospectively enrolled. All the patients underwent esophagogastroduodenoscopy within three months of the enrollment. Unmanipulated images (UI) and Vein-weighted images (VWI) were taken for assessing AWV by a vein-viewing application on iPhone. Two doctors independently evaluated both image types. We defined the grading of both UI and AWV as grade 0 (non-detectable), grade 1 (slightly detectable), and grade 2 (distinct).

\section{Results}

The causes of liver diseases among the 71 men and 29 women (median age, 70.5 year) included Hepatitis $B(n=19)$, Hepatitis $C(n=21)$, alcoholism $(n=3)$, primary biliary cholangitis $(n=4)$, autoimmune hepatitis $(n=4)$ and others $(n=20)$. GOV were indicated in 60 patients, and half of them had not been treated previously (non-treated). VWI could significantly visualize AWV than UI ( $24 \%$ vs $72 \%, p=$ 0.0005 ). The presence of cirrhosis (chronic hepatitis vs cirrhosis $=64.6 \%$ vs $91.4 \%, p=0.004$ ) and GOV $(52.3 \%$ vs $74.3 \%, p=0.032)$ were significantly higher in the VWI-AWV grade 2 group. Multivariate analysis demonstrated that VWI-AWV grade 2 was an independent factor related to the presence of non-treated GOV [OR $=2.97(1.17-7.53), p=0.022]$.

\section{Conclusions}

The vein-viewing application non-invasively detected AWV related to the presence of cirrhosis and GOV, and VWI-AWV grade 2 was an independent factor related to the presence of non-treated GOV.

\section{Background}

Gastroesophageal varices (GOV) are present in about half of the patients with cirrhosis ${ }^{1}$. Variceal bleeding is a life-threatening complication which accounts for $10-30 \%$ of all upper gastrointestinal bleeding ${ }^{2}$. Esophagogastroduodenoscopy (EGD) is the gold standard for the detection of GOV. Disadvantages of endoscopy include the risk of sedation, higher cost, bleeding, and risk of aspiration ${ }^{3}$. 
However, no recommendations on screening of GOV has been made in Japan ${ }^{4}$. Many less invasive methods for screening of GOV have been investigated ${ }^{5}$. Serum biomarkers including platelet count, FIB-4 index, aspartate aminotransferase to platelet ratio index (APRI), liver stiffness (LS), spleen stiffness (SS), LS-spleen diameter to platelet ratio, and Liver stiffness $\times$ spleen size/platelet count (LSPS) are reportedly useful for predicting esophageal varices ${ }^{6-15}$. The updated Baveno VI guidelines recommend that screening EGD can be avoided in patients with compensated advanced chronic liver disease who have liver stiffness $<20 \mathrm{kPa}$ and a platelet count $>150,000 / \mathrm{mm}^{316}$.

We focused on abdominal wall varices (AWV) for predicting GOV. Several prominent collateral veins radiating from the umbilicus are termed the caput-medusae. The caput-medusae sign is an indicator of portal hypertension. It describes engorged paraumbilical veins radiating from the umbilicus within the adipose tissue of the anterior abdominal wall, creating portosystemic anastomoses ${ }^{17}$. However, in clinical settings, it could not be commonly identified. It is now considered a rare finding.

Thus, we found a vein-viewing application for iPhone. This can visualize the high-contrast image of the vein by boosting oxyhemoglobin/deoxyhemoglobin absorption contrast and reducing the contribution of superficially scattered and specularly reflected light to the overall image.

In this study, we aimed to evaluate the efficacy of the vein-viewing application for detecting the AWV in patients with chronic liver disease and elucidating the relationship between AWV and GOV.

\section{Methods}

This was a single-center, prospective, cross-sectional study. Between November 2018 and September 2020 , one-hundred adult patients in our hospital with any chronic liver disease (including cirrhosis) were prospectively enrolled. All the patients underwent EGD within three months of inclusion. Patients with skin diseases of the abdominal wall were not enrolled because of skin discoloration preventing successful imaging. We obtained both unmanipulated images ( $\mathrm{UI})$ and vein-weighted images (VWI) with VeinSeek Pro (VeinSeek LLC, Los Angeles, CA) (https://www.veinseek.com/) for each patient. We defined the grading of AWV as grade 0 (non-detectable), grade 1 (slightly detectable), and grade 2 (distinct) for both unmanipulated and VWI images, respectively (Fig. 1). Both images were evaluated by two doctors (Dr. S and N) independently. We obtained the patient's information on biological gender, age, and mental status (regarding hepatic encephalopathy) at the time of imaging. The following data: total bilirubin, albumin, prothrombin time (PT), fibrosis index based on the four factors (FIB-4) index using age, aspartate transaminase (AST), alanine transaminase (ALT), and platelet values ${ }^{18}$, and AST to platelet ratio index (APRI) ${ }^{19}$ were also collected. The severity of cirrhosis was determined according to the ChildPugh scoring system based on PT, albumin, bilirubin values, and the presence of encephalopathy or ascites. Patients were classified into Child A (5-6 points), B (7-9 points), and C (10-15 points) groups. Classification of GOV was according to the "general rules for recording the endoscopic findings of esophagogastric varices in Japan"20. Other abdominal imaging techniques (ultrasound, computerized 
tomography, or magnetic resonance imaging) were also applied for evaluating ascites. FibroScan measures of liver stiffness were also performed on patients without ascites.

\section{Statistical analysis}

The Student's t-test and chi-square test were applied for comparing the two groups as defined by the cutoff criteria. One-way ANOVA was applied for multiple comparisons. Interrater reliability was assessed by the Cohen's kappa coefficient. A Kappa > 0.7 indicates agreement between two operators. Logistic regression analysis was applied for multivariate analysis. All statistical tests were performed using StatFlex (Windows ver. 6.0; Artech, Osaka, Japan). Values are expressed as median (range) or mean with a standard error of the mean (SEM). Categorical variables are shown as numbers. Statistical significance was set at $p<0.05$.

\section{Results}

\section{Baseline characteristics of the patients}

The baseline characteristics of the patients are presented in Table 1. One-hundred patients [71 men, 29 women, median age, 70.5 (range, 20-87) years] were enrolled in this study. Their liver diseases were induced by the hepatitis $B$ virus (HBV) $(n=19)$, hepatitis $C$ virus $(H C V)(n=21)$, alcohol $(n=33)$, primary biliary cholangitis (PBC) $(n=3)$, autoimmune hepatitis $(A I H)(n=4)$, and others (e.g. Budd-Chiari syndrome and cryptogenic) $(n=20)$. The status of the underlying liver disease was chronic hepatitis in 26 patients and cirrhosis in 74 patients. Cirrhotic patients were classified into Child-Pugh class A $(n=37), B(n=25)$, and $C(n=12)$, respectively. Esophageal varices were detected in 57 of the 100 patients examined and classified into F1 ( $n=26), F 2(n=29)$, and F3 $(n=2)$, respectively. Gastric varices were detected in 24 patients, and the form was classified into F1 $(n=14), F 2(n=7), F 3(n=3)$, respectively. Thirty patients were treated for GOV before enrollment in the study by endoscopic variceal ligation ( $E V L, n=21)$, endoscopic injection sclerotherapy (EIS, $n=6$ ), balloon retrograde transvenous obliteration (B-RTO, n=2), and Hassab's operation $(n=1)$, respectively. Therefore, GOV had been disappeared in three patients at enrollment.

Among the 60 patients with GOV at enrollment, 33 patients had never been treated previously (non-treated group). Portal hypertensive gastropathy (PHG) was identified in 27 patients. Thirty-two patients had ascites. Fifty-nine patients had hepatocellular carcinoma (TNM stage I: II: III: IV = 14: 28: 19: 5).

Encephalopathy was diagnosed in only four patients. Splenomegaly and ascites were found in 32 and 53 patients, respectively.

\section{Table 1. Characteristics of patients}




\begin{tabular}{|c|c|}
\hline Patients & $\mathrm{n}=100$ \\
\hline Sex (Male:Female) & $71: 29$ \\
\hline Age (years) & $70.5(20-87)$ \\
\hline \multicolumn{2}{|l|}{ Etiology of liver disease } \\
\hline HBV infection & 19 \\
\hline HCV infection & 21 \\
\hline Alcoholism & 33 \\
\hline PBC & 3 \\
\hline AlH & 4 \\
\hline Others $^{\dagger}$ & 20 \\
\hline \multicolumn{2}{|c|}{ The status of the underlying liver disease } \\
\hline Chronic hepatitis & 26 \\
\hline Cirrhosis & 74 \\
\hline \multicolumn{2}{|l|}{ Child-Pugh classification } \\
\hline$A: B: C$ & $37: 25: 12$ \\
\hline esophageal varices ${ }^{\ddagger}$ & 57 \\
\hline Location (Li : Lm : Ls) & $10: 34: 13$ \\
\hline Form (F1 : F2 : F3) & $26: 29: 2$ \\
\hline Color (Cw : Cb) & $56: 1$ \\
\hline $\mathrm{RC0}: \mathrm{RC1}: \mathrm{RC2}: \mathrm{RC3}$ & $30: 18: 6: 1$ \\
\hline gastric varicest & 24 \\
\hline Location (Lg-c : Lg-f : Lg-cf) & $13: 10: 1$ \\
\hline Form (F1 : F2 : F3) & $14: 7: 3$ \\
\hline Color (Cw : Cb) & $14: 10$ \\
\hline $\mathrm{RC0}: \mathrm{RC} 1: \mathrm{RC} 2: \mathrm{RC} 3$ & $24: 0: 0: 0$ \\
\hline Past treatment of GOV & 30 \\
\hline EVL : EIS : B-RTO : Hassab & $21: 6: 2: 1$ \\
\hline Past rupture history & 12 \\
\hline Portal hypertensive gastropathy & 27 \\
\hline
\end{tabular}




\begin{tabular}{|c|c|}
\hline Encephalopathy & 4 \\
\hline Ascites & 32 \\
\hline Splenomegaly & 53 \\
\hline
\end{tabular}

tincluding Budd-Chiari syndrome and cryptogenic, $\ddagger$ including treated patients

AlH, autoimmune hepatitis; $\mathrm{Cw}$, white varices; $\mathrm{Cb}$, blue varices; $\mathrm{F} 1$, straight, small-caliber varices; $\mathrm{F}$ 2, moderately enlarged, beady varices; F3, markedly enlarged, nodular or tumor-shaped varices; GOV, gastroesophageal varices; HBV, hepatitis B virus; HCV, hepatitis C virus; Ls, locus superior; Lm, locus medialis; Li. locus inferior; Lg-c, adjacent to the cardiac orifice; Lg-cf, extension from the cardiac orifice to the fornix; PBC, primary biliary cholangitis; RC, red color sign; Data are expressed as median (range).

\section{Abdominal wall varices visualization and classification}

In UI, AWV was classified by two doctors into grade $0(n=72,59)$, grade $1(n=25,33)$, and grade $2(n=3,7)$, respectively. The kappa was 0.5 . In VWI, AWV was classified by two doctors into grade $0(n=16,25)$, grade $1(n=43,30)$, and grade $2(n=41,45)$, respectively. Comparing UI and VWI, the AWV-positive cases (grade1 and 2$)$ were significantly higher in VWI than UI ( $24 \%$ vs $72 \%, p=0.0005)$ (Table 2). In VWI, Grade 0 was decreased, and grade 2 was increased significantly in both doctors compared to the UI grading $(p<0.01)$ (Fig 2). In VWI, the kappa was 0.55 for all grades; however, it was 0.72 for grade 2 classification.

\section{Table 2. Comparison between UI and VWI for depicting AWV}

\begin{tabular}{|llll|}
\hline & VWI-negative & VWI-positive $^{\dagger}$ & total \\
\hline Ul-negative & 28 & 48 & 76 \\
\hline Ul-positive $^{\dagger}$ & 0 & 24 & 24 \\
\hline total & 28 & 72 & 100 \\
\hline
\end{tabular}

Ul, unmanipulated image; VWI, vein-weighed image; AWV, abdominal wall varices; Ul-positive and VWIpositive cases are classified as grade 1 and 2 by two doctors.

\section{Comparing factors between negative and positive GOV}

The comparison factors between negative and positive GOV demonstrated that the presence of cirrhosis was higher in the positive GOV group $(42.5 \%$ vs $95 \%, p<0.001)$. The presence of GOV was significantly higher in patients classified as VWI-AWV grade 2 by both doctors $(22.5 \%$ vs. $43.3 \%, p=0.032)$. For nontreated GOV $(n=70)$, the presence of GOV was also significantly higher in the patients classified as VWIAWV grade 2 by both doctors ( $27.1 \%$ vs. $53.3 \%, p=0.012)$. VWI could also detect grade 2 AWV in eight patients with no varices on their abdomen in UI (Fig. 3). In these eight patients, five patients (63\%) had F2 esophageal varices and RC1 in two. Splenomegaly ( $35 \%$ vs $65 \%, p=0.003)$, VWI-AWV grade $2(22.5 \%$ vs 
$43.3 \%, p=0.032)$, FIB-4 index ( $3.8 \pm 2.5$ vs. $6.1 \pm 4.2, p=0.003)$, APRI $(1.0 \pm 1.0$ vs. $1.6 \pm 1.2, p=0.010)$, and liver stiffness ( $17.0 \pm 16.7$ vs. $28.0 \pm 17.5 \mathrm{kPa}, p=0.011)$ were significantly higher in the positive group. In contrast, platelet count $\left(151.7 \pm 58.5 \times 10^{3}\right.$ vs. $\left.107.7 \pm 49.7 \times 10^{3} / \mathrm{mm}^{3}, p<0.001\right)$, albumin $(3.9$ \pm 0.7 vs. $3.6 \pm 0.6 \mathrm{~g} / \mathrm{dL}, p=0.037)$, and PT ( $84.7 \pm 23.0$ vs. $73.3 \pm 22.2 \%, p=0.017)$ were significantly lower in the positive group (Table 3).

Table 3. Comparison of factors for prediction between patients with or without gastroesophageal varices.

\begin{tabular}{|c|c|c|c|}
\hline & \multicolumn{3}{|c|}{ Gastroesophageal varices $†$} \\
\hline & negative & positive & $p$ value \\
\hline & $(n=40)$ & $(n=60)$ & \\
\hline Gender (male/female) & $27 / 13$ & $44 / 16$ & 0.529 \\
\hline Age (years) & $72(50-87)$ & $69(20-87)$ & 0.050 \\
\hline Child-Pugh scores & $7.2 \pm 2.3$ & $7.1 \pm 2.2$ & 0.885 \\
\hline Splenomegaly (yes / no ) & $14 / 26$ & $39 / 21$ & 0.003 \\
\hline UI-AWV $\left(\right.$ grade0 $/$ grade $\left.\geq 1^{\ddagger}\right)$ & $33 / 7$ & $43 / 17$ & 0.214 \\
\hline UI-AWV (grade0-1 / grade $2^{\ddagger}$ ) & $40 / 0$ & $57 / 3$ & 0.273 \\
\hline VWI-AWV (grade0 / grade $\geq 1^{\ddagger}$ ) & $14 / 26$ & $14 / 46$ & 0.203 \\
\hline VWI-AWV (grade0-1 / grade $2^{\ddagger}$ ) & $31 / 9$ & $34 / 26$ & 0.032 \\
\hline Platelet count $\quad\left(10^{3} / \mathrm{mm}^{3}\right)$ & $151.7 \pm 58.5$ & $107.7 \pm 49.7$ & $<0.001$ \\
\hline FIB4 index & $3.8 \pm 2.5$ & $6.1 \pm 4.2$ & 0.003 \\
\hline APRI & $1.0 \pm 1.0$ & $1.6 \pm 1.2$ & 0.010 \\
\hline Liver stiffness $\S \quad(\mathrm{kPa})$ & $17.0 \pm 16.7$ & $28.0 \pm 17.5$ & 0.011 \\
\hline
\end{tabular}

APRI, aspartate aminotransferase to platelet ratio; AWI, abdominal wall varices; FIB-4, fibrosis index based on the four factors; UI, unmanipulated image; VWI, vein-weighed image; PT, Percent prothrombin time;

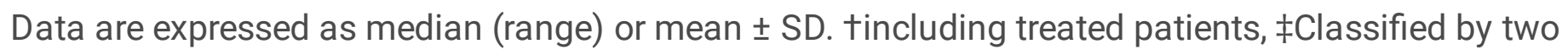
doctors, § Liver stiffness was evaluated in 68 patients without ascites.

\section{Comparing factors between grade $2 \mathrm{AWV}$ and the others of VWI.}

The comparison of factors between VWI-AWV grade 2 and the other grade groups demonstrated that the presence of cirrhosis (CH vs LC $=3 / 32, p=0.004)$, GOV ( $52.3 \%$ vs $74.3 \%, p=0.032)$, ascites $(24.6 \%$ vs $47.1 \%, p=0.023)$, and PHG (20\% vs $40 \%, p=0.023)$ were significantly higher in the VWI-AWV grade 2 
group. In contrast, platelet count $\left(133.7 \pm 60.2 \times 10^{3}\right.$ vs $\left.109.7 \pm 48.9 \times 10^{3} / \mathrm{mm}^{3}, p=0.046\right)$ was significantly lower in the VWI-AWV grade 2 group (Table 4 ).

Table 4. Comparison of factors between patients with VWI-AEV grade 2 and the others

\begin{tabular}{|c|c|c|c|c|}
\hline & \multicolumn{2}{|l|}{ VWI-AWV } & \multirow[t]{3}{*}{$p$ value } \\
\hline & & & Grade $2^{\dagger}$ & \\
\hline & & $(n=65)$ & $(n=35)$ & \\
\hline \multicolumn{2}{|c|}{ Gender (male/female) } & $45 / 20$ & $26 / 9$ & 0.595 \\
\hline \multicolumn{2}{|l|}{ Age (years) } & $71(46-87)$ & $69(20-87)$ & 0.290 \\
\hline \multicolumn{2}{|c|}{ chronic hepatitis / cirrhosis } & $23 / 42$ & $3 / 32$ & 0.004 \\
\hline \multicolumn{2}{|c|}{ Child-Pugh scores } & $6.25 \pm 21.7$ & $7.5 \pm 2.4$ & 0.020 \\
\hline \multicolumn{2}{|c|}{ Ascites (yes / no) } & $16 / 49$ & $17 / 18$ & 0.015 \\
\hline \multicolumn{2}{|c|}{ Splenomegaly (yes / no) } & $32 / 33^{\ddagger}$ & $21 / 14$ & 0.303 \\
\hline \multicolumn{2}{|l|}{ GOV (yes / no) } & 34 / 31 & $26 / 9$ & 0.032 \\
\hline \multicolumn{2}{|c|}{ GOV > F2 (yes / no) } & $19 / 46$ & 14 / 21 & 0.170 \\
\hline \multicolumn{2}{|c|}{ GOV > RC1 (yes / no) } & $13 / 52$ & 12 / 23 & 0.116 \\
\hline \multicolumn{2}{|c|}{ Past treatment of GOV (yes / no) } & $17 / 48$ & $13 / 22$ & 0.253 \\
\hline \multicolumn{2}{|c|}{ Past rupture history (yes / no) } & $5 / 60$ & $7 / 28$ & 0.071 \\
\hline \multicolumn{2}{|l|}{ PHG (yes / no) } & $13 / 52$ & $14 / 21$ & 0.036 \\
\hline Platelet count & $\left(10^{3} / \mathrm{mm}^{3}\right)$ & $133.7 \pm 60.2$ & $109.7 \pm 48.9$ & 0.046 \\
\hline AST & $(U / L)$ & $35.2 \pm 17.6$ & $46.0 \pm 27.6$ & 0.019 \\
\hline ALT & $(U / L)$ & $27.9 \pm 14.5$ & $33.2 \pm 18.4$ & 0.118 \\
\hline Albumin & $(g / d L)$ & $3.8 \pm 0.7$ & $3.5 \pm 0.6$ & 0.053 \\
\hline Total bilirubin & $(\mathrm{mg} / \mathrm{dL})$ & $1.1 \pm 0.7$ & $2.2 \pm 4.5$ & 0.050 \\
\hline PT & $(\%)$ & $80.0 \pm 21.0$ & $73.4 \pm 26.1$ & 0.180 \\
\hline FIB4 index & & $4.9 \pm 4.1$ & $5.7 \pm 3.2$ & 0.302 \\
\hline APRI & & $1.3 \pm 1.2$ & $1.6 \pm 1.0$ & 0.129 \\
\hline Liver stiffness§ & $(\mathrm{kPa})$ & $23.1 \pm 18.1$ & $24.0 \pm 18.0$ & 0.849 \\
\hline
\end{tabular}


AST, aspartate aminotransferase; ALT, alanine aminotransferase; APRI, aspartate aminotransferase to platelet ratio; F2, moderately enlarged, beady varices; FIB4, fibrosis index based on the four factors; GOVs, Gastroesophageal varices; PHG, portal hypertensive gastropathy; PT, Percent prothrombin time; RC, red color sign; Data are expressed as median (range) or mean \pm SD. † Classified by two doctors, $\ddagger$ Two patients had been undergone splenectomy, § Liver stiffness was evaluated in 68 patients without ascites.

\section{Multivariate analysis of predicting factors for GOV.}

Multivariate analysis was applied for the factors related to GOV in Table 3. Liver stiffness was obtained in only 68 patients because of ascites. Therefore, multivariate analysis was applied to groups with $(n=68)$ and without $(n=32)$ liver stiffness data. In the group with liver stiffness, age $\geq 71$ years [OR $=0.28(0.08$ $0.97), p=0.045]$ and VWI-AWV grade2 [OR=3.92 (1.00 - 15.34), $p=0.049$ ] were independent factors. In the group without liver stiffness, only age $\geq 71$ years [OR $=0.29(0.12-0.77), p=0.012$ ] was an independent factor. In this study, liver cirrhosis was lower in patients $\geq 71$ years old (64\% vs. $84 \%, p=$ 0.023). Therefore, age was negatively related to GOV (Table 5).

Table 5. Multivariate analysis of predicting factors for GOV with and without liver stiffness. 


\begin{tabular}{|c|c|c|c|c|}
\hline \multirow[t]{2}{*}{ Factors } & & \multicolumn{3}{|c|}{ Multivariate analysis } \\
\hline & & Odds ratio & $95 \% \mathrm{Cl}$ & $p$-value \\
\hline \multicolumn{5}{|c|}{ with liver stiffness } \\
\hline Age & $\geq 71$ years $^{\dagger}$ & 0.28 & $0.08-0.97$ & 0.045 \\
\hline Splenomegaly & & 2.21 & $0.49-9.81$ & 0.296 \\
\hline Platelet count & $<80,000 / \mathrm{mm}^{3} \ddagger$ & 6.72 & $0.85-53.17$ & 0.071 \\
\hline APRI & $\geq 1.3 \S$ & 0.11 & $0.02-1.57$ & 0.114 \\
\hline FIB-4 index & $\geq 3.23^{9}$ & 2.27 & $0.44-11.78$ & 0.331 \\
\hline Liver stiffness & $\geq 20 \mathrm{kPa}^{*}$ & 1.77 & $0.40-7.76$ & 0.450 \\
\hline VWI-AWV & grade $2^{\star *}$ & 3.92 & $1.00-15.34$ & 0.049 \\
\hline \multicolumn{5}{|c|}{ without liver stiffness } \\
\hline Age & $\geq 71$ years $^{\dagger}$ & 0.29 & $0.12-0.77$ & 0.012 \\
\hline Splenomegaly & & 2.19 & $0.79-6.08$ & 0.133 \\
\hline Platelet count & $<80,000 / \mathrm{mm}^{3 \ddagger}$ & 3.03 & $0.62-14.70$ & 0.170 \\
\hline APRI & $\geq 1.3^{\S}$ & 0.45 & $0.09-2.17$ & 0.316 \\
\hline FIB-4 index & $\geq 3.23^{9}$ & 2.44 & $0.73-8.11$ & 0.145 \\
\hline VWI-AWV & grade $2^{*}$ & 2.45 & $0.89-6.78$ & 0.084 \\
\hline
\end{tabular}

APRI, aspartate aminotransferase to platelet ratio; AWI, abdominal wall varices; FIB-4, fibrosis index based on the four factors; GOV, Gastroesophageal varices; VWI, vein-weighed image.taccording to the median age of all the patients, faccording to the report by Burton et $\mathrm{al}^{21}, \S$ according to the report by Castéra et $\mathrm{al}^{22} \uparrow$ according to the report by Kraja et al ${ }^{23}$ * according to the updated Baveno $\mathrm{VI}$ guidelines ${ }^{16},{ }^{*}$ Classified by two doctors.

\section{Multivariate analysis of factors for non-treated GOV.}

Multivariate analysis was also applied for non-treated GOV. It was also applied both with and without liver stiffness. Only VWI-AWV grade2 was an independent factor related to non-treated GOV in both individuals with [OR $=3.43(1.03-11.44), p=0.045]$ and without [OR $=2.97(1.17-7.53), p=0.022]$ liver stiffness data (Table 6).

Table 6. Multivariate analysis of predicting factors for non-treated GOV with liver stiffness. 


\begin{tabular}{|c|c|c|c|c|}
\hline \multirow[t]{2}{*}{ Factors } & & \multicolumn{3}{|c|}{ Multivariate analysis } \\
\hline & & Odds ratio & $95 \% \mathrm{Cl}$ & $p$-value \\
\hline \multicolumn{5}{|c|}{ with liver stiffness } \\
\hline Age & $\geq 71$ years $^{\dagger}$ & 0.49 & $0.14-1.77$ & 0.276 \\
\hline Splenomegaly & & 1.30 & $0.30-5.69$ & 0.729 \\
\hline Platelet count & $<80,000 / \mathrm{mm}^{3} \ddagger$ & 3.15 & $0.53-18.82$ & 0.208 \\
\hline APRI & $\geq 1.3 \S$ & 0.85 & $0.11-6.58$ & 0.874 \\
\hline FIB-4 index & $\geq 3.23^{9}$ & 0.62 & $0.11-3.57$ & 0.593 \\
\hline Liver stiffness & $\geq 20 \mathrm{kPa}^{*}$ & 0.78 & $0.18-3.44$ & 0.744 \\
\hline VWI-AWV & grade $2^{* *}$ & 3.43 & $1.03-11.44$ & 0.045 \\
\hline \multicolumn{5}{|c|}{ without liver stiffness } \\
\hline Age & $\geq 71$ years $^{\dagger}$ & 0.66 & $0.26-1.67$ & 0.380 \\
\hline Splenomegaly & & 0.79 & $0.26-2.37$ & 0.675 \\
\hline Platelet count & $<80,000 / \mathrm{mm}^{3 \neq}$ & 1.59 & $0.42-6.05$ & 0.497 \\
\hline APRI & $\geq 1.3^{\S}$ & 1.49 & $0.36-6.15$ & 0.578 \\
\hline FIB-4 index & $\geq 3.23^{9}$ & 0.79 & $0.21-2.94$ & 0.729 \\
\hline VWI-AWV & grade $2^{*}$ & 2.97 & $1.17-7.53$ & 0.022 \\
\hline
\end{tabular}

APRI, aspartate aminotransferase to platelet ratio; AWI, abdominal wall varices; FIB-4, fibrosis index based on the four factors; GOV, Gastroesophageal varices; VWI, vein-weighed image. taccording to the median age of all the patients, łaccording to the report by Burton et $\mathrm{al}^{21}{ }^{21}, \S$ according to the report by Castéra et $\mathrm{al}^{22} \uparrow$ according to the report by Kraja et al ${ }^{23}$ * according to the updated Baveno $\mathrm{VI}$ guidelines ${ }^{16},{ }^{*}$ Classified by two doctors.

\section{Discussion}

In this pilot study, we demonstrated that the vein-viewing application on iPhone could non-invasively detect AWV related to cirrhosis and GOV. This is the first report of the non-invasive method of simply taking AWV images that enables us to indicate the patients who should be applied for medical service and EGD. 
Among the forty patients without GOV the mean platelet count was over $150,000 / \mathrm{mm}^{3}$, and mean liver stiffness was $17 \mathrm{kPa}$. This suggested that the cutoff levels for avoiding EGD in the Baveno VI guidelines were practical in indicating patients with a low risk of GOV.

In our study, age was an independent factor negatively related to GOV. This was an unexpected result. The difference in etiology may have caused this. The number of patients with alcoholism was larger in the participant group under 71 years of age compared to the older group ( $42 \%$ vs. $24 \%, p=0.056)$. Moreover, most of the patients with alcoholism had cirrhosis (87.9\%).

The image-based method for the prediction of GOV has been validated. Further development will enhance the usefulness of this approach in future medical diagnostics. Smartphones and mobile devices have rapidly become part of everyday life around the world. In the current situation with COVID-19 the role of on-line medical services is increasingly important. The vein-viewing application on the iPhone was not originally developed for medical purposes; however, we have established that it is useful in detecting AWV in cirrhotic patients in a medical context.

In this study, VWI-AWV grade 2 was related to the presence of cirrhosis, high Child-Pugh score, the presence of ascites, the presence of GOV, the presence of PHG, and low platelet count. Furthermore, multivariate analysis demonstrated that VWI-AWV grade 2 was an independent factor related to nontreated GOV. GOV treatment would alter the hemodynamics, including AWV. Our approach is therefore more meaningful for diagnosing naïve than treated patients. Intriguingly, eight patients $(22.9 \%)$ who were identified as grade 2 had no AWV when assessed by Ul. Five patients with GOV (three were untreated) were included in the eight patients. The interrater reliability was lower in VWI-AWV grade $0-1$, indicating that identifying a slight AWV was difficult. However, the identification of grade 2 AWV was significantly higher by VWI in both doctors, and the reliability of VWI-AWV grade 2 was satisfactory.

Among twenty-two VWI-AWV grade 2 patients who had no history of GOV treatment, six patients did not have any GOV. In this group, four patients (67\%) had cirrhosis. VWI-AWV grade 2 may therefore have the potential to identify not only GOV but also cirrhosis. However, the other two patients had no cirrhosis and GOV; this would be an entirely false positive. Novel technology is warranted for the improvement of the vein-viewing application to minimize this outcome.

The role of artificial intelligence is also rapidly growing in the medical field, such as pathology, EGD, mammography, brain diseases, and COVID-19 diagnosis ${ }^{24-28}$. Deep learning of AWV structures would provide a highly reproducible diagnosis of AWV. It also means that each person can check themselves with such applications on mobile devices in the future. Our effort should be focused on quantifying the imaging capabilities of mobile devices on the human body and provide meaning and context to them.

This study has several limitations. The cohort studied represented a small group of patients on which EGD could be performed. Selection bias was therefore inevitable. However, based on the promising results of this pilot study, a large-scale cohort study will be conducted for validation. 
In summary, the vein-viewing application could non-invasively detect AWV related to the presence of cirrhosis and GOV. VWI-AWV grade 2 was an independent factor related to the presence of non-treated GOV. This result suggests a future direction of medicine using consumer mobile devices as medical devices. The camera lens will be like the eyes on "Baymax," a prototype healthcare-providing robot on Disney animation.

\section{List Of Abbreviations}

\section{AlH}

autoimmune hepatitis

APRI

aspartate aminotransferase to platelet ratio

AST

aspartate aminotransferase

ALT

alanine aminotransferase

AWI

abdominal wall varices

B-RTO

balloon retrograde transvenous obliteration

EGD

esophagogastroduodenoscopy

EVL

endoscopic variceal ligation

EIS

endoscopic injection sclerotherapy

FIB-4

fibrosis index based on the four factors

GOV

gastroesophageal varices

HBV

hepatitis $B$ virus

HCV

hepatitis $C$ virus

MRI

magnetic resonance of imaging

PBC

primary biliary cholangitis

PHG

portal hypertensive gastropathy 
percent prothrombin time

RC

red color sign

Ul

unmanipulated image

VWI

vein-weighed image

\section{Declarations}

Ethics approval and consent to participate: The study protocol was approved by the Institutional Review Board of our institute (No. 18A152) under the guidelines of the 1975 Declaration of Helsinki. This study was registered in UMIN-CTR (http://www.umin.ac.jp/ctr/index-j.htm), identification number (R000040890). Written informed consent was obtained from all patients.

Consent for publication: The consent for publication has been obtained from all the patients.

Availability of data and materials: The data that support the findings of this study are available from the corresponding author, Sugihara T., upon reasonable request.

Competing interests: The authors declare that they have no competing interests.

Funding: None

Authors' contributions: H.Y., S.T., S.I., M.Y., and N.T. designed and carried out the experiment. S.T. wrote the manuscript with support from O.J., and I.H. supervised the project.

Acknowledgements: None

\section{References}

1. Nusrat S, Khan MS, Fazili J, Madhoun MF. Cirrhosis and its complications: Evidence based treatment. World J Gastroenterol. 2014;20:5442-60.

2. LaBrecque D, Khan AG, Sarin SK, LeMair AW. Esophageal varices. World Gastroenterology Organisation Global Guidelines; 2014

3. Philips CA, Sahney A. Oesophageal and gastric varices: Historical aspects, classification and grading: Everything in one place. Gastroenterol Rep. 2016;4:186-95.

4. Fukui H, Saito H, Ueno Y, Uto H, Obara K, Sakaida I, et al. Evidence-based clinical practice guidelines for liver cirrhosis 2015. J Gastroenterol. 2016;51:629-50.

5. Karatzas A, Konstantakis C, Aggeletopoulou I, Kalogeropoulou C, Thomopoulos K, Triantos C. Noninvasive screening for esophageal varices in patients with liver cirrhosis. Ann Gastroenterol. 
2018;31:305-14.

6. Chalasani N, Imperiale TF, Ismail A, Sood G, Carey M, Wilcox CM, et al. Predictors of large esophageal varices in patients with cirrhosis. Am J Gastroenterol. 1999;94:3285-91.

7. Zaman A, Hapke R, Flora K, Rosen HR, Benner K. Factors predicting the presence of esophageal or gastric varices in patients with advanced liver disease. Am J Gastroenterol. 1999;94:3292-6.

8. Gue CS, Yap CK, Ng HS. The correlation between cytopenia and esophageal varices in patients with liver cirrhosis. Med J Malaysia. 2004;59:604-8.

9. Deng H, Qi X, Guo X. Diagnostic accuracy of APRI, AAR, FIB-4, FI, king, lok, forns, and fibroindex scores in predicting the presence of esophageal varices in liver cirrhosis. Medicine (Baltimore). 2015;94:e1795.

10. De Franchis R, Dell'Era A. Invasive and noninvasive methods to diagnose portal hypertension and esophageal varices. Clin Liver Dis. 2014;18:293-302.

11. Ma X, Wang L, Wu H, Feng $Y, \operatorname{Han} X, B u H$, et al. Spleen stiffness is superior to liver stiffness for predicting esophageal varices in chronic liver disease: A meta-analysis. PLoS One. 2016;11:1-15.

12. Shin SU, Lee JM, Yu MH, Yoon JH, Han JK, Choi BI, et al. Prediction of esophageal varices in patients with cirrhosis: Usefulness of three-dimensional MR elastography with echo-planar imaging technique. Radiology. 2014;272:143-53.

13. Colecchia A, Montrone L, Scaioli E, Bacchi-Reggiani ML, Colli A, Casazza G, et al. Measurement of spleen stiffness to evaluate portal hypertension and the presence of esophageal varices in patients with HCV-related cirrhosis. Gastroenterology. 2012;143:646-54.

14. Shibata S, Joshita S, Umemura T, Yamazaki T, Fujimori N, Ichikawa Y, et al. Liver stiffness-spleen size-to-platelet ratio risk score detects esophageal varices in chronic liver disease. Springerplus. 2016;5:1-6.

15. Berzigotti A, Bosch J, Boyer TD. Use of noninvasive markers of portal hypertension and timing of screening endoscopy for gastroesophageal varices in patients with chronic liver disease. Hepatology. 2014;59:729-31.

16. De Franchis R; Baveno VI Faculty. Expanding consensus in portal hypertension: Report of the Baveno VI Consensus Workshop: Stratifying risk and individualizing care for portal hypertension. J Hepatol. 2015;63:743-52.

17. Giambelluca D, Caruana G, Cannella R, Picone D, Midiri M. The "caput medusae" sign in portal hypertension. Abdom Radiol. 2018;43:2535-36.

18. Vallet-Pichard A, Mallet V, Nalpas B, et al. FIB-4: An inexpensive and accurate marker of fibrosis in HCV infection. Comparison with liver biopsy and FibroTest. Hepatology. 2007;46:32-6.

19. Wai CT, Greenson JK, Fontana RJ, Kalbfleisch JD, Marrero JA, Conjeevaram HS, et al. A Simple noninvasive index can predict both significant fibrosis and cirrhosis in patients with chronic hepatitis C. 2003;38:518-26. 
20. Miyaaki H, Ichikawa T, Taura N, Miuma S, Isomoto H, Nakao K. Endoscopic management of esophagogastric varices in Japan. Ann Transl Med. 2014;2:1-6.

21. Burton JR, Liangpunsakul S, Lapidus J, Giannini E, Chalasani N, Zaman A. Validation of a multivariate model predicting presence and size of varices. J Clin Gastroenterol. 2007;41:609-15.

22. Castéra L, Le Bail B, Roudot-Thoraval F, Bernard PH, Foucher J, Merrouche W, et al. Early detection in routine clinical practice of cirrhosis and oesophageal varices in chronic hepatitis $\mathrm{C}$ : Comparison of transient elastography (FibroScan) with standard laboratory tests and non-invasive scores. J Hepatol. 2009;50:59-68.

23. Kraja B, Mone I, Akshija I, Koçollari A, Prifti S, Burazeri G, et al. Predictors of esophageal varices and first variceal bleeding in liver cirrhosis patients. World J Gastroenterol. 2017;23:4806-14.

24. Bera K, Schalper KA, Rimm DL, Velcheti V, Madabhushi A. Artificial intelligence in digital pathology new tools for diagnosis and precision oncology. Nat Rev Clin Oncol. 2019;16:703-15.

25. Hajjar A El, Rey JF. Artificial intelligence in gastrointestinal endoscopy: General overview. Chin Med J (Engl). 2020;133:326-34.

26. Kim HE, Kim HH, Han BK, Kim KH, Han K, Nam HE, et al. Changes in cancer detection and falsepositive recall in mammography using artificial intelligence: a retrospective, multireader study. Lancet Digit Heal. 2020;2:e138-48.

27. Rauschecker AM, Rudie JD, Xie L, Wang J, Gee JC. Neuroradiologist-level differential diagnosis accuracy at brain MRI. Radiology. 2020;295:626-37.

28. Zhang R, Tie X, Qi Z, Bevins NB, Zhang C, Griner D, Song TK, et al. Diagnosis of COVID-19 pneumonia using chest radiography: Value of artificial intelligence. Radiology 2020;202944.

\section{Figures}




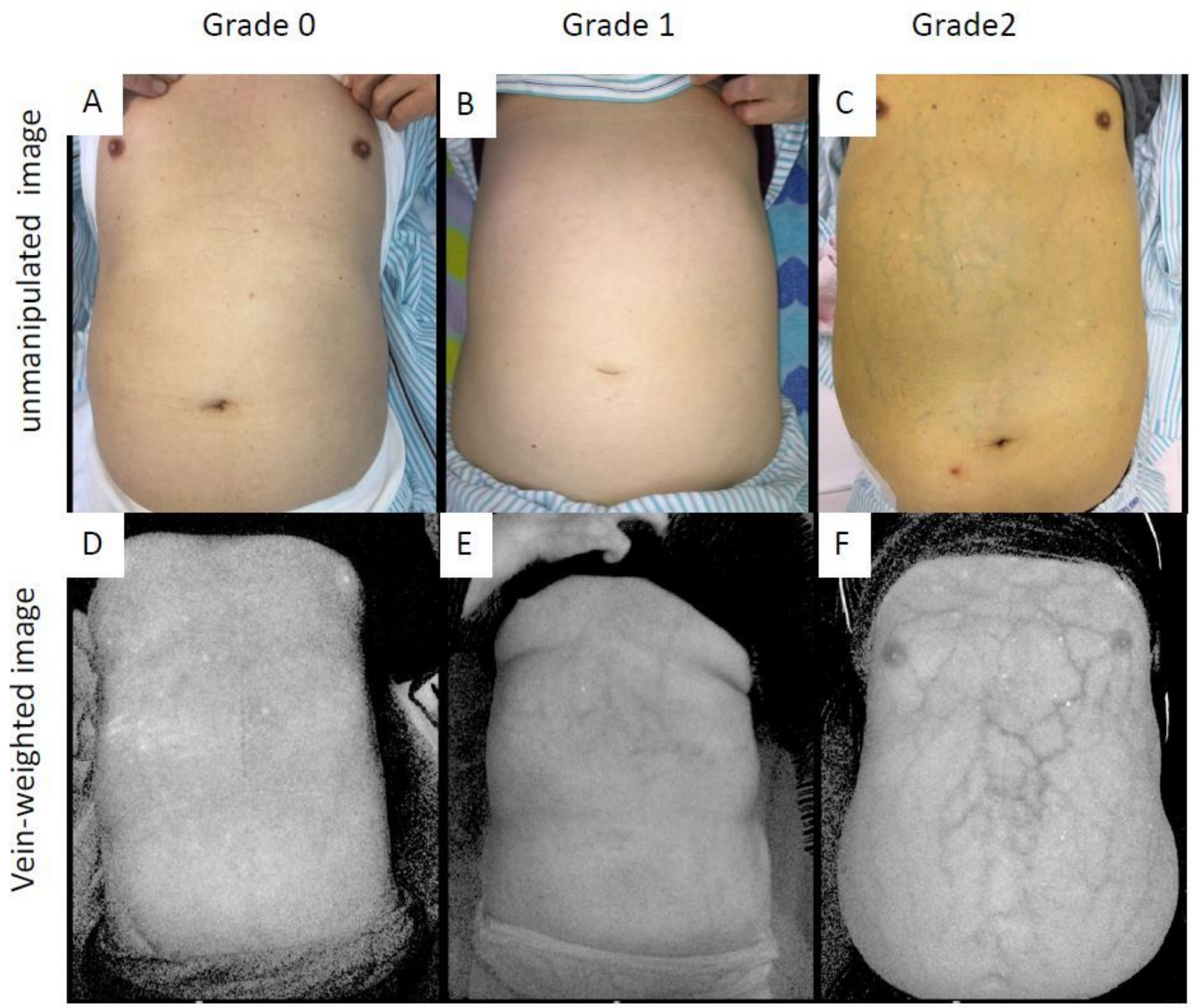

Fig.1

\section{Figure 1}

Classification of abdominal wall varices (A) grade0; UI-non-detectable, (B) grade1; Ul-slightly detectable, (C) grade2; UI-distinct, (D) grade0; VWI-non-detectable, (E) grade1; VWI-slightly detectable, (F) grade2; VWIdistinct, UI, Unmanipulated image; VWI, Vein-weighted image 


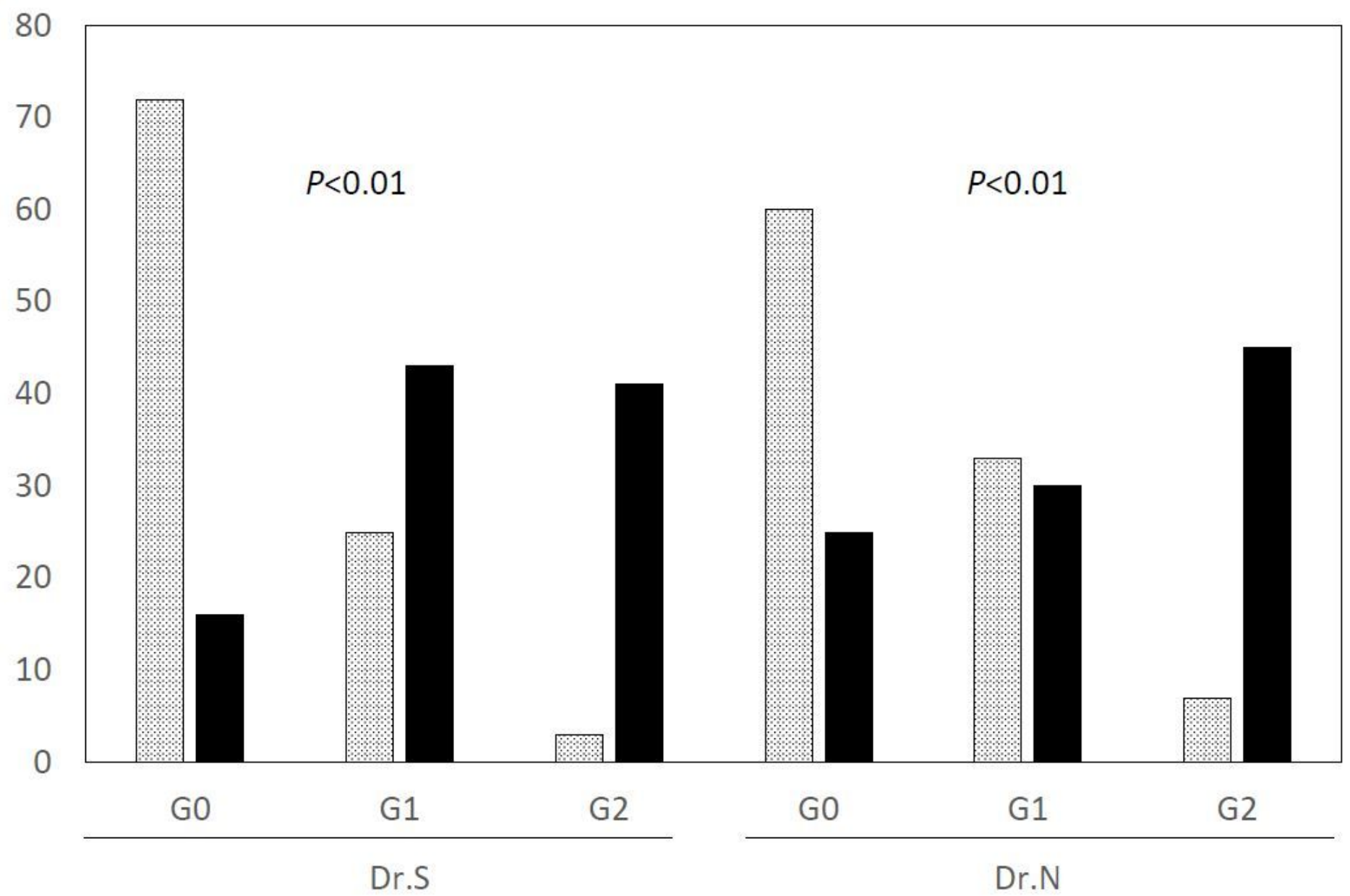

Fig.2

Figure 2

Classification of abdominal wall varices by two doctors Grade 0 was decreased in VWI, and grade 2 was increased in VWI in both doctors. UI, Unmanipulated image; VWI, Vein-weighted image 


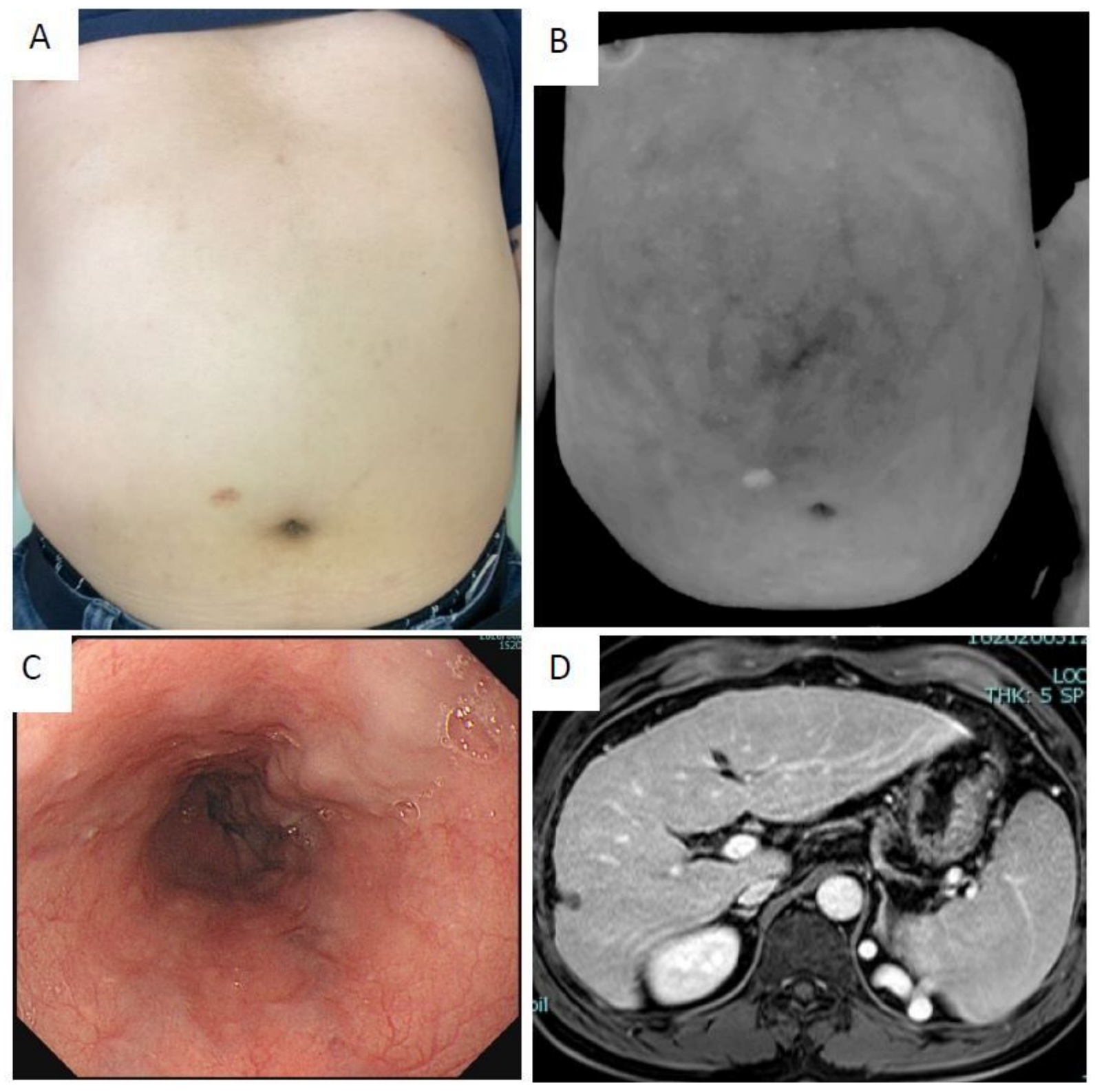

Fig.3

Figure 3

A representative case of VWI-AWV grade 2 whose UI-AWV was grade 0 . (A) UI-AWV image classified grade 0, (B) VWI-AWV image classified grade 2, (C) Esophageal varices classified F2, (D) MRI showed cirrhosis and splenomegaly AWV, abdominal wall varices; MRI, magnetic resonance of imaging; UI, Unmanipulated image; VWI, Vein-weighted image 